\title{
High Expression of HMOX1 in Blue-Shelled Chickens is Associated with a TG Haplotype
}

\section{-Author(s)}

Wang Z'

Deng X"

Wang A ${ }^{\prime \prime \prime}$

Liu $R^{\prime}$

College of Animal Science and Technology, Northwest A\&F University, Yangling 712100, China

" National Engineering Laboratory for Animal Breeding and the Key Laboratory of Animal Genetics and Breeding of the Ministry of Agriculture, China Agricultural University, Beijing, 100193, China

III State Key Laboratory of Direct-Fed Microbial Engineering, Beijing 100192, China

\section{Mail Address}

Corresponding author e-mail address Ruifang Liu

College of animal science and technology, Northwest A\&F University, Xinong Road, 22, Yangling, Shaanxi, 712100, China. Phone/Fax: +86 (029) 87092102

E-mail: liuruifang79@163.com

\section{nKeywords}

Chicken, blue egg, biliverdin, HMOX1, differential expression.

\section{ABSTRACT}

HMOX 1 is an important gene in biosynthesis of the eggshell pigment of blue eggs. Previous studies found that HMOX1 is highly expressed in the shell gland of hens laying blue eggs $(\mathrm{BlueH}) \mathrm{compared}$ with hens laying brown eggs (BrownH); however, the reasons for the differential expression are unclear. In this study five single nucleotide polymorphism (SNP) in HMOX1 were genotyped in $111 \mathrm{BlueH}$ and $115 \mathrm{BrownH}$. The association of haplotypes of these SNP with the blue egg phenotype was tested. Haplotype-specific expression of HMOX1 was detected in the shell gland. The interaction of sequence variants and transcription factors was analyzed using electrophoretic mobility shift assay (EMSA). A TG haplotype covering upstream $1.4 \mathrm{~kb}$ region of HMOX1 was significantly associated with blue eggs $(p<0.05)$. Furthermore, the birds $(n=12)$ with the haplotype expressed 3.8 fold more transcripts than those $(n=12)$ without the haplotype $(p<0.05)$. After re-sequencing a $2.2 \mathrm{~kb}$ region harboring the TG haplotype, a total of 26 SNP were found, of which a SNP was predicted to create a binding site of Nrf2, a transcription factor initiating HMOX 1 expression. However, subsequent EMSA failed to confirm the Nrf2-DNA interaction. Taken together, the data suggested that the TG haplotype is not directly involved in regulation of HMOX1 expression; a regulatory mutation located near the haplotype and linked with the haplotype may exist and be responsible for the differential expression of HMOX1.

\section{INTRODUCTION}

Birds are the only class of vertebrates that have evolved eggshell pigmentation (Cassey et al., 2010). The eggshell colors have been proposed to serve a wide variety of visual, physical, physiological, and behavioral functions, such as cryptic coloring, resisting harmful solar radiation, thermal protection, reinforcing eggshell strength, sexual selection, etc. (Reynolds et al., 2009). Thus, appearance of eggshell colors represents an important event of bird evolution. Blue-green coloration is a common eggshell color among birds. It is present not only in wild birds, but also in domestic fowls (i.e., several indigenous chicken breeds, such as Chilean Araucano, Chinese Dongxiang, and Lushi chickens) (Kennedy \& Verves, 1973; Wang et al., 2013). The blue-green coloration is believed to function as a signal of female health to attract males involved in the supervision of the offspring (Morales et al., 2010).

Blue egg is colored by biliverdin, a bile pigment derived from the oxidative degradation of heme (Lang \& Well, 1987). Two studies have confirmed that hens laying blue eggs (BlueH) have significant higher levels of biliverdin in eggshell and shell gland than those laying brown eggs (BrownH) (Zhao et al., 2006; Wang et al., 2009). However, molecular mechanisms underlying this difference are poorly understood. Blue-green eggshell color, also called as oocyan, is genetically controlled by a dominant mutant (O) (Punnett, 1933). In the latest study, Wang 
et al. (2013) identified the $O$ as a retrovirus (EAV$\mathrm{HP}$ ) insertion in SLCO1B3. Because OATP1B3 (gene protein) is a membrane transporter that can mediate transportation of biliverdin (Hagenbuch \& Gui 2008), it is speculated that formation of blue egg may be relevant to abnormal transportation of the eggshell pigment in shell gland (Wang et al., 2013).

In addition of SLCO1B3, two studies found higher HMOX1 expression in the shell glands of $\mathrm{BlueH}$ than of BrownH (Wang et al., 2010; Wang et al., 2011). Because heme oxygenase-1 ( $\mathrm{HO}-1$, protein encoded by $H M O X 1)$ is the rate-limiting enzyme catalyzing oxidative degradation of heme into biliverdin (Maines, 1997), the above result implied that HMOX1 can play a crucial role in biosynthesis of eggshell biliverdin.

The aim of this study was to further explore the regulatory mechanisms underlying high expression of HMOX 1 in the shell glands of BlueH. The association of HMOX 1 haplotype with blue egg phenotype was tested in the groups of BlueH and BrownH. Haplotype-specific expression of $H M O X 1$ was detected in the shell glands. The effect of sequence variants on HMOX1 expression was studied using electrophoretic mobility shift assay.

\section{MATERIAL AND METHODS}

\section{Birds and Sample Collection}

In this study, Dongxiang chickens were used to explore how the regulatory mechanisms of HMOX1 expression work. Dongxiang is a well-known blue egglaying breed from Dongxiang city, Jiangxi province, China. Although blue egg phenotype has been specifically selected in the Dongxiang chickens, the trait has not been fixed in the group, in which most birds lay blue-green eggs, but there are still minor individuals laying light brown eggs. A total of $111 \mathrm{BlueH}$ and 115
BrownH were used for haplotype association analysis. All birds were provided by Hualv Dongxiang chicken protection farm, Jiangxi province, China.

Twelve birds with TG haplotype (TG_) and 12 birds without the haplotype $(X X)$ were euthanized approximately three to five $h$ prior to ovulation. Shell glands were collected and immediately stored in liquid nitrogen for subsequent expression analysis. Use and care of birds in this study was approved by the Northwest A\&F University Ethics Committee.

\section{SNP genotyping and haplotype analysis}

Five SNPs were used to construct the HMOX1 haplotype. These SNPs, covering over $7 \mathrm{~kb}$ of the HMOX1 genome sequence, are sufficient to capture most of the variation in HMOX1, as assessed using the Tagger program of Haploview 4.2 (Broad Institute, Cambridge, MA). Information on the SNPs is summarized in Table 1.

Blood samples were collected by wing vein puncture. Genomic DNA was extracted from the blood using a standard phenol-chloroform method. The SNPs were genotyped by polymerase chain reaction-restriction fragment length polymorphism. Restriction enzymes were summarized in Table 1. All fragments were resolved on $2.5 \%$ agarose gels or $12 \%$ nondenaturing polyacrylamide gels and visualized after an ethidium bromide or sliver staining.

All allele frequencies were tested for confirmation with the Hardy-Weinberg equilibrium. Construction and association analysis of the haplotype were completed using Haploview v4.2 under default values. Haplotypes with frequencies lower than 0.01 were discarded. In "sliding windows" analysis, 2-SNP, 3-SNP, 4-SNP window sizes were used to detect whether

Table 1 - Information on the five SNPs used in HMOX1 haplotype association

\begin{tabular}{|c|c|c|c|c|c|c|}
\hline Name & dbSNP Accession $\mathrm{No}^{1}$ & Allele & $\begin{array}{l}\text { SNP positions in } \\
\text { UCSC }^{2}\end{array}$ & $\begin{array}{l}\text { SNP positions in } \\
\text { HMOX1 }\end{array}$ & primer sequence $\left(5^{\prime}-3^{\prime}\right)$ & Restriction enzyme \\
\hline HSNP1 & rs314037822 & $\mathrm{G} / \mathrm{T}$ & Chr1:51956353 & $5^{\prime}$ upstream region & $\begin{array}{l}\text { F: AGCTCCAGATGGAAGAGG } \\
\text { R: GGTGATAGAGGAGGACAGC }\end{array}$ & $\operatorname{Hinf} \mathrm{I}$ \\
\hline HSNP2 & rs315838673 & $A / G$ & Chr1:51954906 & intron1 & $\begin{array}{l}\text { F: CGCTCCTCCTCTCACCTCTG } \\
\text { R: GCCGTGCTGTTACACCCTAC }\end{array}$ & Sau I \\
\hline HSNP3 & rs313454440 & $\mathrm{G} / \mathrm{A}$ & Chr1:51954831 & $\begin{array}{l}\text { exon2, synonymous } \\
\text { variant }\end{array}$ & $\begin{array}{l}\text { F: GACCGGGAGGAGAAGCATGG } \\
\text { R: ACACGGCTCTGGAACACC }\end{array}$ & Sacl \\
\hline HSNP4 & rs3137366 & $\mathrm{T} / \mathrm{C}$ & Chr1:51952346 & exon3, V122A & $\begin{array}{l}\text { F: GCAGACAAAAGACACACC } \\
\text { R: GCTGTAACCCACAACTTC }\end{array}$ & HPY99I \\
\hline HSNP5 & rs13866562 & $A / G$ & Chr1: 51949356 & 3'UTR & $\begin{array}{l}\text { F: ACTGGGCTCTGCCACTAATA } \\
\text { R:GAGCAAACAGAGCTCCGGGAGGTGCA }\end{array}$ & ApaL I \\
\hline
\end{tabular}

${ }^{1}$ dbSNP database: http://www.ncbi.nlm.nih.gov/snp

2 SNP positions refer to galGal4 assembly of UCSC database (http://genome .ucsc.edu).

${ }^{3}$ The reverse primer for rs 13866562 was modified to create a specific sequence of restriction enzyme of $A p a L$ I. The modified base is indicated in bold. 
some regions can carry stronger association with blue egg than others.

\section{Haplotype-specific expression analysis}

Total RNA was extracted with TRIzol reagent (TianGen Co., Beijing, China). Two micrograms of total RNA were reverse transcribed in a final volume of $25 \mu \mathrm{L}$, containing $1 \mu \mathrm{L}$ of $20 \mu \mathrm{M}$ Oligo (dT)15 primer, $5 \mu \mathrm{L}$ of first-strand buffer $(250 \mathrm{mM}$ Tris- $\mathrm{HCl}$, $375 \mathrm{mM} \mathrm{KCl}, 15 \mathrm{mM} \mathrm{MgCl}_{2}, 50 \mathrm{mM}$ DTT), $5 \mu \mathrm{L}$ of $10 \mathrm{mM}$ dNTP, $1 \mu \mathrm{L}$ of $25 \mathrm{U}$ Recombinant RNasin $\circledast$ Ribonulease Inhibitor, $1 \mu \mathrm{L}$ of $200 \mathrm{U} / \mu \mathrm{L}$ M-MLV reverse transcriptase (Promega, Wisconsin, USA), and RNasefree water to final volume.

Real-time PCR was performed in an ABI PRISM 7900 instrument (Applied Biosystems Corp., Foster, CA). Amplification of $1 \mu \mathrm{L}$ of cDNA was carried out in a total volume of $20 \mu \mathrm{L}$, according to the RealMasterMix (SYBR Green I) manual (Tiangen Corp., Beijing, China). Every sample was evaluated in triplicate. Data were analyzed by the $2^{\wedge}-\triangle \triangle C T$ method (Livak \& Schmittgen, 2001). The XX group was designed as calibrator. GAP$D H$ was used as endogenous reference to normalize the amount of CDNA added to the PCR. The amount of $H M O X 1$ transcripts, normalized to GAPDH and relative to the calibrator, was given by $2^{\wedge}-\triangle \triangle C T$. The forward and reverse primers were $5^{\prime}$-ATCGCATGAAAACAGTCCAG-3' and 5'-CAAATAAGCCCACGGCGAC-3' for HMOX1 and 5'-ATACACAGAGGACCAGGTTG-3' and 5'-AAACTCATTGTCATACCAGG-3' for GAPDH.

\section{Electrophoretic Mobility Shift Assays (EMSA)}

The two biotin-labeled oligonucleotides used in EMSA reaction were 5'-CGCGGCTCTCTGCTCTICCTCCTGCTGCCCCCGG-3' and 5'-CGCGGCTCTCTGCTCTAACTCCTGCTGCCCCCGG-3', corresponding to two alleles (shown in bold and underlined letters) of TC and AA at TSNP21, respectively. Nuclear proteins were extracted from the shell glands using the Nuclei Protein Extraction Kit (Viagene Biotech Inc, Beijing, China), following the manufacturer's protocol. Protein concentration was determined with the BCA Protein Assay Kit (Cwbio Co. Beijing, China). Five $\mu \mathrm{g}$ of nuclear proteins and $2.5 \mathrm{ng}$ of biotin-labeled probes were incubated at room temperature for $20 \mathrm{~min}$ in binding mixture containing $1.5 \mu \mathrm{L}$ of $10 \times$ binding buffer and $1 \mu \mathrm{g}$ of poly $(\mathrm{dl}) \bullet \operatorname{poly}(\mathrm{dC})$ in a total volume of $15 \mu \mathrm{L}$. In antibody supershift assays, 2 $\mu \mathrm{g}$ of Anti-Nrf2 rabbit polyclonal antibody (Abcam Co., Shanghai, China) were added to the reaction mixture and incubated for $20 \mathrm{~min}$ at room temperature prior to electrophoresis. In competition assays, firstly a 100-fold molar excess of unlabeled oligonucleotides was add- ed to the binding mixture, then incubated for $30 \mathrm{~min}$ at room temperature. Secondly, labeled probes were added and subjected to additional 20 min incubation at room temperature. DNA-protein complexes were resolved on a $5 \%$ nondenaturing polyacrylamide gel in $0.5 \times$ TBE at $80 \mathrm{~V}$ for 2-3 h. After electrophoresis, the complexes were transferred to a membrane and detected with horseradish peroxidase-conjugated streptavidin. Finally, blots were visualized using EMSA Chemiluminescent system (Viagene Biotech Inc, Beijing, China).

\section{Statistical analysis}

For comparison of HMOX 1 expression between birds with TG haplotype and those without the haplotype, the null hypothesis that there was no difference between two groups was tested by unpaired $t$ test. Statistical significance was assumed at $p<0.05$. All statistical analyses were done by SAS ${ }^{\circledR}$ V8.2 statistical software package (PC-SAS) (Cary, NC, USA).

\section{RESULTS}

\section{Association of HMOX1 haplotypes with blue egg}

In order to elucidate whether there are sequence variants responsible for high expression of HMOX1 in shell gland of BlueH, the association of HMOX1 haplotypes, consisting of 5 SNPs, with blue egg phenotype was analyzed. Five major haplotypes were observed in BlueH and BrownH, of which only the GAGTG haplotype showed a significant association with blue eggs $(p<0.05$, Table 2$)$. In order to explore whether some regions of HMOX1 can carry a stronger association, 4-, 3- and 2-SNP "sliding window" analysis were performed. As the "sliding window" size was reduced, three haplotypes (TGGT, TGG, and TG) showed increasing association with blue egg, with the TG haplotype showing the strongest association with blue eggs (Table 3).

Table 2 - Frequencies of haplotypes constructed by the five SNPS

\begin{tabular}{lccc}
\hline \multirow{2}{*}{ Haplotype $^{1}$} & \multicolumn{2}{c}{ Frequencies } & $\begin{array}{c}\text { Chi-square } P \\
\text { value }\end{array}$ \\
\cline { 2 - 3 } GGACA & BrwonH $^{3}$ & BlueH $^{3}$ & 0.9571 \\
TAGTA & 0.218 & 0.216 & 0.8696 \\
TAGCG & 0.183 & 0.177 & 0.1704 \\
GAGCA & 0.113 & 0.158 & 0.0578 \\
GAGTG $^{2}$ & 0.139 & 0.082 & $\mathbf{0 . 0 2 1 2}$ \\
all rare<0.05 & $\mathbf{0 . 0 5 0}$ & $\mathbf{0 . 1 0 7}$ & 0.0936 \\
\hline
\end{tabular}

${ }^{1}$ Only haplotypes with frequencies $\geq 0.05$ are shown. Rare haplotypes with frequencies $<0.05$ were combined.

${ }^{2} \mathrm{~A}$ significant result is highlighted in bold.

${ }^{3}$ BrownH $=$ hens laying brown eggs, BlueH = hens laying blue eggs 
Table 3 - Results of 2, 3 and 4-SNP "sliding window" analysis

\begin{tabular}{lcccc}
\hline \multirow{2}{*}{ Configurations } & \multirow{2}{*}{ Haplotype $^{1}$} & \multicolumn{2}{c}{ Frequencies } & \multirow{2}{*}{$\begin{array}{c}\text { Chi-square } \\
\text { P value }\end{array}$} \\
\cline { 3 - 4 } & & BrownH $^{2}$ & BlueH $^{2}$ & \\
\hline HSNP2-HSNP3-HSNP4-HSNP5 & GGTG & 0.010 & 0.039 & 0.0432 \\
\hline HSNP2-HSNP3-HSNP4-HSNP5 & GGCA & 0.007 & 0.039 & 0.0206 \\
\hline HSNP2-HSNP3-HSNP4 & GGC & 0.006 & 0.040 & 0.017 \\
\hline HSNP1-HSNP2-HSNP3-HSNP4 & TGGT & 0.025 & 0.063 & 0.0489 \\
\hline HSNP1-HSNP2-HSNP3 & TGG & 0.026 & 0.077 & 0.0133 \\
\hline HSNP1-HSNP2 & TG & 0.029 & 0.099 & 0.0022 \\
\hline
\end{tabular}

${ }^{1}$ Only haplotypes significantly associated with blue egg are shown in the table.

${ }^{2}$ BrownH $=$ hens laying brown eggs, $\mathrm{BlueH}=$ hens laying blue eggs

\section{Haplotype-specific expression analysis}

The TG haplotype covers upstream $1.4 \mathrm{~kb}$ region of HMOX1, which is traditionally a key region for the regulation of gene expression. A haplotype-specific expression assay was performed using hens birds $(n=12)$ with the TG haplotype (TG_) and hens $(n=12)$ without the haplotype $(X X)$ to investigate the role of the haplotype in the regulation of HMOX1 expression. The expression data showed that the $\mathrm{TG}_{-}$group expressed 3.8-fold more transcripts than the XX group in shell gland $(p<0.05$, Figure 1).

Figure 1 - Haplotype-specific expression analysis in shell gland

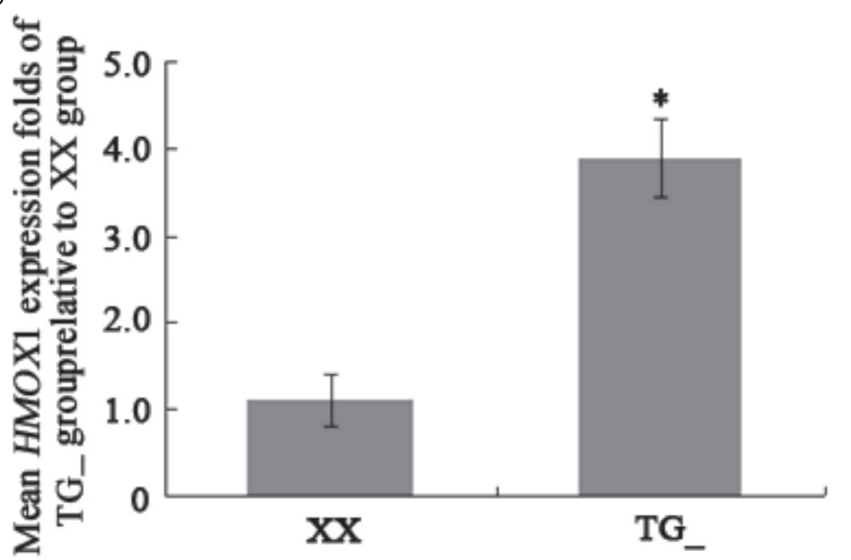

TG_ group consisted of birds $(n=12)$ with the TG haplotype, correspondingly XX for ones $(n=12)$ without the haplotype. The results were presented as mean folds of HMOX1 expression relative to the XX group. The TG group expressed approximate 3.8 fold more transcripts than the $X X$ group $\left({ }^{*} p<0.05\right)$.

\section{Effects of sequence variants within the TG-covering region on transcription factor- DNA interactions}

A $2.2 \mathrm{~kb}$ region of Chr1:51954712-51956937, harboring the TG haplotype, was re-sequenced using $10 \mathrm{BlueH}$ and $10 \mathrm{BrownH}$ to identify crucial regulatory variants. A total of 26 SNPs were found in the region, most of which showed significant association with blue eggs (Table 4). After performing a prediction of transcription factor binding sites (TFBS) using CONSITE software (http://consite.genereg.net/) (LENHARD et al. 2003), 11 SNPs of these SNPs were found to create a putative TFBS (Table 4), with special attention paid to TSNP21 because the blue egg-associated TC allele of the TSNP21 can create a putative TFBS of Nrf2. It has been well established that Nrf2 is a transcription factor initiating HMOX1 expression under oxidative stress (Campbell et al., 2013).

An EMSA assay, therefore, was performed, using nuclear extracts from the shell gland and probes corresponding to $\mathrm{TC}$ and $\mathrm{AA}$ alleles in order to investigate the authenticity of Nrf2-mediated expression. Similar shifted bands were detected in lanes 2 and 6 with TC and AA probes, suggesting that there was no obvious difference in transcription factorbinding activity between two TSNP21 alleles (Figure 2). After performing a supershift assay with anti-Nrf2 antibody, we also failed to observe supershifted bands in lanes 3 and 7 (Figure 2), which, therefore, implied that the protein-DNA complexes in lanes 2 and 6 may be produced by the binding of other proteins, instead of $\mathrm{Nrf2}$, to the oligonucleotides.

Figure 2 - EMSA validated Nrf2-TSNP21 interaction

$\begin{array}{rccccccccc}\text { Lane } & 1 & 2 & 3 & 4 & 5 & 6 & 7 & 8 \\ \text { Nuclear extract } & - & + & + & + & - & + & + & + \\ \text { labled TC probe } & + & + & + & + & - & - & - & - \\ \text { labled AA probe } & - & - & - & - & + & + & + & + \\ \text { Cold probe } & - & - & - & + & - & - & - & + \\ \text { Anti-Nrf2 antibody } & - & - & + & - & - & - & + & -\end{array}$

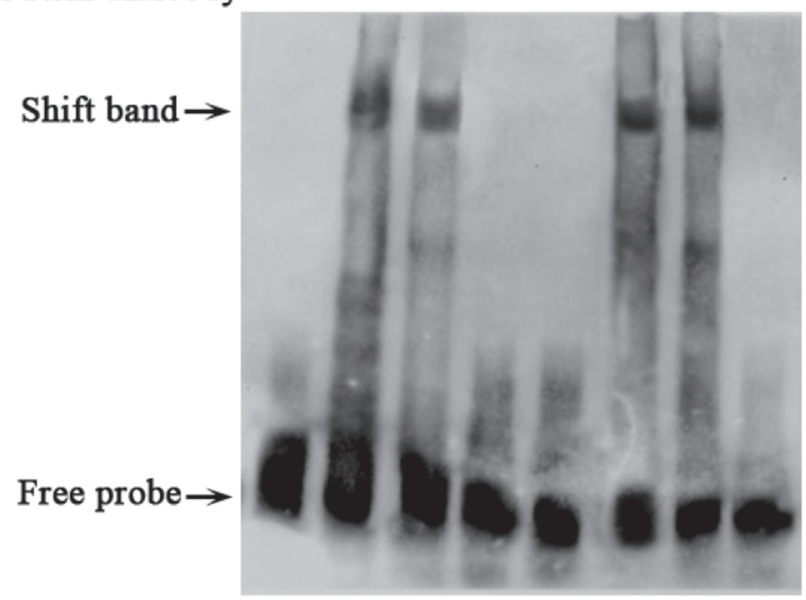

Nrf2 is a well-established transcription factor initiating HMOX1 expression under oxidative stress. A putative Nrf2-binding element was created by the TC allele of the TSNP2 1 locus. EMSA was performed to validate the protein-DNA interaction using nuclear extracts from the shell gland and two probes corresponding to TC and AA alleles of TSNP21. The positions of free probe and shifted DNA-protein complexes are indicated with arrows. In lanes 1 and 5, no shifted band was observed when no nuclear extracts added; in lanes 2 and 6 , the same shifted bands were observed using two probes, indicating that there was no obvious difference in transcription factor-binding activity between two alleles; in lanes 3 and 7, the shifted bands were not supershifted by Nrf2 antibody, indicating that the observed complexes in lanes 3 and 7 can consist of other protein, instead of Nrf2, and oligonucleotides; in lane 4 and 8, the shifted bands can be competed out by $100 \times$ molar excess of unlabelled probes (cold probe), confirming that the complex consists of two target probes and unknown proteins. 
Table 4 - Effect of SNPs within a TG-covering region of Chr1: $51954712-51956937$ on transcript factor binding sites

\begin{tabular}{|c|c|c|c|c|c|c|}
\hline Name & dbSNP accession No. & Position at Chr. $1^{1}$ & Position at HMOX1 & Alleles & Chi-square $\mathrm{P}$ value ${ }^{2}$ & Effect of SNP on TFBS 3 \\
\hline TSNP1 & rs314913481 & 51956882 & & $A / G$ & $p<0.05$ & no \\
\hline TSNP2 & rs312661490 & 51956662 & & A/G & $p<0.05$ & no \\
\hline TSNP3 & new & 51956591 & & $\mathrm{~T} / \mathrm{C}$ & $p<0.05$ & no \\
\hline TSNP4 & new & 51956521 & & $\mathrm{G} / \mathrm{A}$ & $p<0.05$ & no \\
\hline TSNP5 & rs315124462 & 51956427 & & $\mathrm{C} / \mathrm{T}$ & $p<0.05$ & $C \rightarrow T$, FREAC -4 \\
\hline TSNP6 & new & 51956403 & & $\mathrm{~A} / \mathrm{C}$ & $p<0.05$ & $C \rightarrow A$, Thing1-E47 \\
\hline TSNP7 & rs316233291 & 51956396 & & $C / G$ & $p<0.05$ & $G \rightarrow C$, Myf \\
\hline TSNP8 & rs314166379 & 51956380 & & $A / G$ & $p<0.05$ & \\
\hline HSNP1 & rs314037822 & 51956353 & 5' upstream region & $\mathrm{G} / \mathrm{T}$ & $p<0.05$ & \\
\hline TSNP9 & rs315494484 & 51956346 & & $\mathrm{C} / \mathrm{T}$ & $p<0.05$ & \\
\hline TSNP10 & new & 51956248 & & $\mathrm{C} / \mathrm{T}$ & $p<0.05$ & \\
\hline TSNP11 & new & 51956226 & & $C / T$ & $p<0.05$ & \\
\hline TSNP12 & new & 51955536 & & A/G & $p>0.05$ & $\mathrm{G} \rightarrow \mathrm{A}$, Max \\
\hline TSNP13 & new & 51955474 & & $C / T$ & $p>0.05$ & $\mathrm{C} \rightarrow \mathrm{T}, \mathrm{c}-\mathrm{FOS}$ \\
\hline TSNP14 & rs14824170 & 51955419 & & $\mathrm{C} / \mathrm{T}$ & $p>0.05$ & $\mathrm{~T} \rightarrow \mathrm{C}, \mathrm{Spz} 1$ \\
\hline TSNP15 & rs317039830 & 51955410 & & $C / G$ & $p>0.05$ & \\
\hline TSNP16 & new & 51955322 & & $\mathrm{C} / \mathrm{T}$ & $p>0.05$ & $\mathrm{~T} \rightarrow \mathrm{C}, \mathrm{ARNT}$ \\
\hline TSNP17 & rs314520877 & 51955099 & & $\mathrm{~A} / \mathrm{C}$ & $p<0.05$ & \\
\hline TSNP18 & new & 51954974 & & A/G & $p<0.05$ & \\
\hline TSNP19 & new & 51954966 & & $A / G$ & $p<0.05$ & A: AML-1, G: Myf \\
\hline TSNP20 & new & 51954944 & intron1 & $\mathrm{C} / \mathrm{T}$ & $p<0.05$ & $\mathrm{C} \rightarrow \mathrm{T}, \mathrm{Spz} 1$ \\
\hline TSNP21 & new & $\begin{array}{l}51954940- \\
51954941\end{array}$ & & TC/AA & $p<0.05$ & AАХTC, Nrf2 \\
\hline HSNP2 & rs315838673 & 51954906 & & A/G & $p<0.05$ & \\
\hline TSNP22 & rs313881629 & 51954876 & & $\mathrm{~T} / \mathrm{C}$ & $p<0.05$ & \\
\hline TSNP23 & rs313454440 & 51954831 & Exon2, synonymous variant & $A / G$ & $p>0.05$ & \\
\hline TSNP24 & rs314101757 & 51954807 & Exon2, synonymous variant & $\mathrm{A} / \mathrm{G}$ & $p>0.05$ & $\mathrm{G} \rightarrow \mathrm{A}, \mathrm{CHOP}, \mathrm{Nrf2}$ \\
\hline
\end{tabular}

' SNP positions at chromosome1 were given according to chicken genome galGal4 assembly in UCSC database (http://genome.ucsc.edu/cgi-bin/hgGateway).

${ }^{2}$ Association of SNP alleles with blue egg was tested by Chi-square.

${ }^{3} \mathrm{~A}$ putative transcript factor binding sites (TFBS) was created with alternation of alleles, which was predicted using the CONSITE software (http://asp.ii.uib.no:8090/cgi-bin/CONSITE/ consite).

\section{DISCUSSION}

Biliverdin, a bile pigment, previous regarded as a waste product of heme breakdown, actually has an important role in scavenging free radicals (Bulter \& Mcgraw, 2013). In birds, the bile pigment also plays the role of pigmenting the eggshell with blue-green color (Duval et al., 2013). Biliverdin is derived from the oxidative degradation of heme, with the formation of equimolar $\mathrm{CO}$ and $\mathrm{Fe}^{2+}$. In this reaction, heme oxygenase $(\mathrm{HO})$ is the rate-limiting enzyme (Maines, 1997). Previous studies have found that HO-1 (one of three $\mathrm{HO}$ isozymes) have a higher level of expression in shell the gland of BlueH than BrownH (Wang et al., 2010; Wang et al., 2011), which therefore suggested that HO-1 is most likely to play a role in the expression of the blue egg phenotype as well. However, the regulatory mechanism underlying the differential expression remains unclear. This study found a TG haplotype to be significantly associated with blue egg phenotype. Subsequent haplotype-specific expression analysis further revealed that the haplotype has a higher expression activity, which implied that a cis-regulation variant may exist in the HMOX1 and be responsible, or partly at least, for the differential expression of HMOX1 between BlueH and BrownH.

To find out the variant, we sequenced a $2.2 \mathrm{~kb}$ sequence covering the TG haplotype and found a suspicious SNP, which can create a putative binding site of Nrf2. However, EMSA failed to verify the authenticity of the Nrf2-DNA interaction. This urges us to rethink the role of the TG haplotype in regulation of HMOX1 expression. It is plausible that the phenotype-associated haplotype itself is not involved directly in regulation of HMOX1 expression; it may only serve as a marker indicating that an adjacent mutation, situated in linkage disequilibrium with it, may mediate the high HMOX1 expression in BlueH. The inference obtained a support from studies to cis-acting elements of HMOX1. HO- 1 is an inducible cytoprotective protein in the defense system against oxidative stress (Calay \& Mason, 2014). A long list 
of cis-regulatory elements, such as ARE, HeRE, HSE, CdRE, MARE, MPRE, and (GT)n repeats, were found to be involved in regulation of HMOX1 expression in human, rat and chicken (Martin et al., 2004; Chang et al., 2004; Shibahara et al., 1989; Shibahara et al., 2003; Shan et al., 2004). Thus, it is conceivable that any variants in these cis-elements may be responsible for the differential expression as well.

In the case of trans-acting factors three MAPK pathways of ERK, JNK, and p38 are known to be involved in up-regulation of $\mathrm{HO}-1$ expression (Martin et al., 2004). In addition, trans-acting protein BACH1 is a repressor of Nrf2-mediated $\mathrm{HO}-1$ expression. Its inactivation is a prerequisite for $\mathrm{HO}-1$ induction (Reichard et al., 2007). Thus, any function or expression alterations in these trans-regulatory factors can also become the reasons for the differential expression of HMOX1.

Chicken oviduct is a highly vascular organ. When oocytes leave the ovary and enter the oviduct, the metabolism of the oviduct is stimulated into activity; blood flow through it increases; albumen is largely synthesized and secreted (Kennedy \& Ververs, 1973; Baird et al. 1975). Following the process, a large amount of reactive oxygen species will be produced, causing deleterious effects on cellular proteins, lipids, and DNA (Alonso-Alvarez et al., 2004). HO-1 is a crucial protein in resisting oxidative injury (Calay \& Mason, 2014). Thus, its high expression in BlueH may imply that appearance of blue egg is a result of bird adaptive evolution in the defense against oxidative stress during lay.

Based on previous studies of HMOX1, this work attempted further to reveal the reasons for the high expression of the gene in BlueH. Here, a TG haplotype was found to represent high expression activity. Because the haplotype appears more frequently (0.099) in BlueH than in BrownH (0.029), the data explained the reasons for high expression of HMOX1 to some extent. However, given the complex regulatory mechanisms of HMOX1, it remains an enormous challenge to define which of the variants is responsible for its differential expression. It, therefore, is necessary further to explore functional variants in an extended region and several trans-acting factors in the future.

\section{ACKNOWLEDGEMENTS}

This study was supported by the National Natural Science Foundation of China (Grant No. 31401051) and China Postdoctoral Science Foundation (2014M550510).

\section{REFERENCES}

Alonso-Alvarez C, Bertrand S, Devevey C, Prost J, Faivre B, Sorci G. Increased susceptibility to oxidative stress as a proximate cost of reproduction. Ecology Letters 2004;7(5): 363-368.

Baird T, Solomon SE, Tedstone DR. Localization and characterization of eggshell porphyrin in several avian species. British Poultry Science 1975;16(2):201-208.

Bulter MW, Mcgraw KJ. Immune function is related to adult carotenoid and bile pigment levels, but not to dietary carotenoid access during development, in female mallard ducks. Journal of Experimental Biology 2013;216(Pt 14):2632-2640.

Calay D, Mason JC. The multifunctional role and therapeutic potential of $\mathrm{HO}-1$ in the vascular endothelium. Antioxidant \& Redox Signaling 2014;20(11):1789-1809.

Campbell MR, Karaca M, Adamski KN, Chorley BN, Wang XT, Bell DA. Novel hematopoietic target genes in the NRF2-mediated transcriptional pathway. Oxidative Medicine and Cellular Longevity 2013;2013(2013):1-12.

Cassey P, Portugal SJ, Maurer G, Ewen JG, Boulton RL, Hauber ME, Blackburn TM. Variability in avian eggshell colour: a comparative study of museum eggshells. PLoS ONE 2010;5(8):e12054.

Chang KW, Lee TC, Yeh WI, Chung MY, Liu CJ, Chi LY, Lin SC. Polymorphism in heme oxygenase-1 ( $\mathrm{HO}-1)$ promoter is related to the risk of oral squamous cell carcinoma occurring on male areca chewers. British Journal of Cancer 2004;91(8):1551-1555.

Duval C, Cassey P, Mikšík I, Reynolds SJ, Spencer KA. Condition-dependent strategies of eggshell pigmentation: an experimental study of Japanese quail (Coturnix coturnix japonica). Journal of Experimental Biology 2013;216(Pt4):700-708

Hagenbuch B, Gui C. Xenobiotic transporters of the human organic anion transporting polypeptides (OATP) family. Xenobiotica 2008;38(78):778-801

Kennedy GY, Ververs HG. Eggshell pigments of the Araucano fowl. Comparative Biochemistry and Physiology B-Biochemistry \& Molecular Biology 1973;44(1):11-25.

Lang MR, Well JW. A review of eggshell pigmentation. Worlds Poultry Science Journal 1987;43(3):238-246

Lenhard B, Sandelin A., Mendoza L, Engstrom P, Jareborg N, Wasserman WW. Identification of conserved regulatory elements by comparative genome analysis. Journal of Biology 2003;2(2):13.

Livak KJ, Schmittgen TD. Analysis of relative gene expression data using real-time quantitativem PCR and the 2(-Delta Delta $C(T)$ ) method. Methods 2001;25(4):402-408.

Maines MD. The heme oxygenase system: a regulator of second messenger gases. Annual Review of Pharmacology and Toxicology 1997;37:517554.

Martin D, Rojo Al, Salinas M, Diaz R, Gallardo G, Alam J, et al. Regulation of heme oxygenase-1 expression through the phosphatidylinositol 3-kinase/Akt pathway and the Nrf2 transcription factor in response to the antioxidant phytochemical carnosol. Journal of Biological Chemistry 2004;279(10):8919-8929.

Morales J, Torres R, Velando A. Parental conflict and blue egg coloration in a seabird. Naturwissenschaften 2010;97(2):173-180.

Punnett RC. Genetic study in poultry-IX. The blue egg. Journal of Genetics $1933 ; 27(3): 465-470$. 
Reichard JF, Motz GT, Puga A. Heme oxygenase-1 induction by NRF2 requires inactivation of the transcriptional repressor $\mathrm{BACH} 1$. Nucleic Acids Research 2007:35(21):7074-7086.

Reynolds SJ, Martin GR, Cassey P. Is sexual selection blurring the functional significance of eggshell coloration hypotheses? Animal Behaviour 2009;78(1):209-215

Shan Y, Lambrecht RW, Bonkovsky HL. Identification of key elements that are responsible for heme-mediated induction of the avian heme oxygenase-1 gene. Biochimica et Biophysica Acta-Gene Structure and Expression 2004;1679(2):87-94

Shibahara S, Sato M, Muller RM, Yoshida T. Structural organization of the human heme oxygenase gene and the function of its promoter. European Journal of Biochemistry 1989;179(3):557-563.

Shibahara S, Nakayama M, Kitamuro T, Udono-Fujimori R, Takahashi K. Repression of heme oxygenase-1 expression as a defense strategy in humans. Experimental Biology and Medicine 2003;228(5):472-473.
Wang XT, Zhao CJ, Li JY, Xu GY, Lian LS, Wu CX, Deng XM. Comparison of the total amount of eggshell pigments in Dongxiang brownshelled eggs and Dongxiang blue-shelled eggs. Poultry Science 2009;88(8):1735-1739.

Wang XT, Zhao CJ, Li JY, Xu GY, Lian LS, Wu CX, Deng XM. Heme Oxygenase-1 is important to the formation of eggshell biliverdin in chicken. Journal of Applied Animal Research 2010;38(2):229-232.

Wang ZP, Liu RF, Wang AR, Li JY, Deng XM. Expression and activity analysis reveal that heme oxygenase (decycling) 1 is associated with blue egg formation. Poultry Science 2011;90(4):836-841.

Wang ZP, Qu LJ, Yao JF, Yang XL, Li GQ, Zhang YY, et al. An EAV-HP insertion in 5' flanking region of SLCO1B3 causes blue eggshell in the chicken. PLoS Genetics 2013;9(1):e1003183.

Zhao R, Xu GY, Liu ZZ, Li JY, Yang N. A study on eggshell pigmentation: biliverdin in blue-shelled chickens. Poultry Science 2006;85(3):546-549. 
